\title{
Skin Infections
}

\author{
Nancy Rihana and Mindy Sampson
}

\begin{abstract}
Cutaneous infections are common in immunocompromised patients. Neutropenia predisposes patients to fungal, bacterial and viral infections. Antibacterial antifungal and antiviral prophylaxis have caused a significant reduction in some of these infections.

There are two main types of cutaneous infections: primary cutaneous infections and cutaneous manifestations of a disseminated infection. In the latter, skin lesions may be the window to disseminated bloodstream infection and the first and only evidence of a disseminated life threatening infection.

The diagnosis may be at your fingertips; therefore a thorough skin exam is the clue. However, it's also important to know the characteristic lesions associated with different infections. It will help expedite diagnosis so appropriate treatment is initiated promptly in neutropenic patients, which can be lifesaving.

In a retrospective study of 43 neutropenic febrile patients with cutaneous lesions, fungal infections were the most frequent, and nodular lesions on the lower extremities were the most prevalent (Naorungroj and Aiempanakit, J Am Acad Dermatol 74:AB166, 2016).
\end{abstract}

Skin biopsy for pathological study and culture remains the gold standard and should be obtained early to confirm the suspected diagnosis. In these immunocompromised patients the inflammatory response is altered by either the primary disease or its treatment. Therefore, routine pathogens may present in an atypical fashion, with diminished or absent induration, erythema, or pustulation in response to bacterial resulting cutaneous infection without typical cellulitis (Urabe, Clin Infect Dis 39:S53-S55, 2004). Skin lesions are evaluated not only by morphology, but also in the context of the clinical setting and biopsy result. The skin biopsy is inexpensive, relatively noninvasive and without contraindication, and may avoid the need for more invasive procedures such an open lung biopsy (Grossman, et al., Cutaneous manifestations of infection in the immunocompromised host. Springer Science+Business Media, LLC, New York, 2012).

N. Rihana $(\varangle) \cdot$ M. Sampson

University of South Florida, Tampa, FL, USA

e-mail: nrihana@health.usf.edu 
In addition to antimicrobial therapy, surgery should not be postponed in the face of progressive skin and soft tissue infection in this population (Brzozowski and Ross, J Hand Surg Br 22:679-680, 1997).

Keywords Ecthyma gangrenosum $\cdot$ Bullous cellulitis $\cdot$ Fulminant necrotizing infections · Spontaneous clostridial myonecrosis · Purplish discoloration · Crepitation $\cdot$ Necrotizing enterocolitis $\cdot$ Subcutaneous nodules $\cdot$ Disseminated cutaneous mycobacterium $\cdot$ knife-cut sign $\cdot$ Herpetic whitlow $\cdot$ Disseminated HZ

\section{Bacterial Infections}

\section{Gram Negative Bacteria}

Neutropenia is one of the major risk factors for gram-negative cellulitis. We will review the main gram negative pathogens and their cutaneous manifestations in this population.

Pseudomonas aeruginosa, usually a nosocomial pathogen has emerged as a common cause of infection in immunocompromised patients; most often in neutropenic leukemics during chemotherapy. The routine use of anti-pseudomona antimicrobial prophylaxis in cancer patients with prolonged neutropenia has reduced the overall incidence of pseudomonal infections. However the emergence fluroquinolone resistant gram negatives including Pseudomonas remains an important threat in neutropenic patients. Pseudomonas can invade through areas of micro erosions especially over intravenous, urinary catheters, decubital ulcerations and thermic damage [1].

Cutaneous lesions occur in $30 \%$ of cases of Pseudomonas bacteremia [2]. The site of origin of the Pseudomonas is most commonly the respiratory or genitourinary tract. P. aeruginosa infection can also be acquired from a humid environment including showers, sinks, and flower vases.

The dermatologic manifestations of Pseudomonas sepsis include ecthyma gangrenosum, hemorrhagic bullae, necrotizing/gangrenous or bullous cellulitis, painful vesicular lesions, and small papules on the trunk resembling rose spots of typhoid fever, grouped petechiae, erysipelas-like lesions with hyperesthesia, erythematous or violaceous subcutaneous painful nodules, and necrotizing or malignant external otitis [3].

Ecthyma gangrenosum (EG) has classically been considered a pathognomonic sign of Pseudomonas aeruginosa septicemia, although it can be reported with multiple other gram-negative bacterial, fungal, and viral infections in the immunocompromised host. It can occur as single or multiple lesions. Most cases of ecthyma gangrenosum have been associated with septicemia, but it can also occur in the absence of Pseudomonas bacteremia. The most common sites for ecthyma gangrenosum are the gluteal, perineal area, axillary and extremities [4]. 
It begins as an area of erythema and edema that progresses to hemorrhagic bullae that rupture, evolving into a painless central blackish gray necrotic area (eschar) surrounded by an erythematous halo. It grows up to several centimeters in diameter over 12-24 h. The necrosis may extend as deep as muscle. Pus is minimal. Lesions at various stages of development may be present at different sites in the same patient [3]. Ulcerations are sensitive on palpation [1]. It is assumed that necrosis of the skin is caused by Pseudomonas elastase which destroys elastic lamina of the blood vessels and allows liberation of the pathogen into the subcutaneous tissues [5] (Fig. 1).

The pathologic hallmark of EC is vasculitis sparing the intima and without thrombosis which distinguishes it from other forms of bacterial vasculitis in which septic intraluminal thrombi attach to bacteria and invade the endothelium [4].

E. Coli, is a common cause of cellulitis in the immunocompromised host that is impossible to distinguish from streptococcal cellulitis. It is rapidly progressive and limb threatening if appropriate antibiotic are not started promptly [3,6]. E. coli may also produce abscesses anywhere in the body but mainly cause perirectal phlegmon in neutropenic patients.

Ecthyma gangrenosum described above, is considered pathognomonic for Pseudomonas aeruginosa but has also been described with multiple other gramnegative organisms including E. coli, E. cloacae, Klebsiella, Serratia, Citrobacter, Morganella, and Stenotrophomonas [3, 7-11]

Aeromonas species are gram-negative, non-sporulating facultative anaerobic bacillus found in brackish or fresh water lakes, streams and soils. They have also been recovered from chlorinated tap water, including hospital water supplies. It may be isolated as well from stools of asymptomatic carriers. Among patients with cancer, Aeromonas septicemia is more likely to occur in leukemic patients and its associated with water related activities in only $10 \%$ cases but is nosocomially acquired in $60 \%$ [12]. About $20-30 \%$ of infections are associated with skin lesions.

In immunosuppressed patients, Aeromonas can cause various infections that are likely to be fatal including severe cellulitis, fulminant necrotizing infections, and ecthyma gangrenosum. Myonecrosis and gas production have occurred with Aeromonas and can simulate clostridial gas gangrene [3].

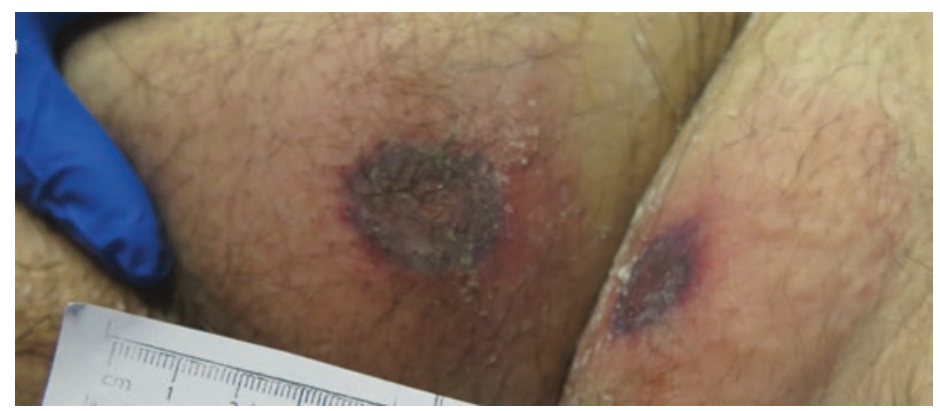

Fig. 1 Ecthyma gangrenosum secondary to Pseudomonas aeruginosa in a neutropenic patient after induction therapy for acute myelogeneous leukemia 
Aeromonas infections of freshwater traumatic wounds after water related activities such as fishing or swimming cause a rapidly progressive cellulitis that develops within $8-48 \mathrm{~h}$ after trauma followed by suppuration and necrosis around the wound, myonecrosis and sometimes osteomyelitis. It often requires surgical debridement in addition to antibiotic therapy [4].

Chromobacterium violaceum is a rare but frequently fatal infection. Its an anaerobic gram-negative rod that is commonly found in soil and water in tropical and sub-tropical climates between latitudes $35^{\circ}$ north and $35^{\circ}$ south. Cutaneous infection is rare and usually occurs with exposure of broken or injured skin to contaminated muddy or stagnant water or soil in patients with neutrophil dysfunction or HIV infection. Infection in the skin tends to present with cellulitis, pustules, ulcers, or abscesses accompanied by severe systemic symptoms [3].

\section{Gram Positive Bacteria}

Over the course of the last several decades, the frequency with which gram-positive bacteria have been isolated from neutropenic patients with cancer has increased [13]. We will review the main gram positive pathogens that lead to cutaneous involvement in neutropenic patients.

Bacillus cereus commonly presents in a neutropenic patient as a single painful vesicle, pustule, or bulla on a digit or extremities with rapidly spreading cellulitis during the spring and summer. The bulla may become necrotic and develop a black eschar. It's usually associated with systemic toxicity [3]. Gram stain of the aspirate smear demostrates large Gram-positive rods, which may be mistaken for Clostridium infection and treated with penicillin. B. cereus and B subtilus cause most of the infections. About half of these infections arise at sites of indwelling intravascular catheters [4]. occasionally widely disseminated blood stream infections may occur including endocarditis and brain abscess [14-16].

Clostridium species account for $30 \%$ of anaerobic bacteremias in all cancer patients [17]. It tends to cause a fulminant infection in neutropenic patients. Sixty percent of the isolated cases are secondary to Clostridium perfringens and $30 \%$ by Clostridium septicum. Clostridium perfringens is usually part of the colonic flora while C. septicum is typically found in soil and animals, but is not normal human flora [3]. Forty percent of the clostridium infections are polymicrobial, which indicates the gastrointestinal tract as the source of these infections [17].

Two types of cutaneous manifestation have been described in neutropenic patients: spontaneous, non-traumatic gas gangrene, and spreading cellulitis [4].

(a) Spontaneous clostridial myonecrosis (gas gangrene) most commonly associated with a silent colon carcinoma, an underlying hematologic tumor or neutrophil dysfunction [3]. Its is clinically characterized by the sudden onset of excruciating pain in the involved site- usually the leg esp. if associated with abdominal involvement. The swelling is rapidly progressive, associated with purplish/ bronze discoloration and blister formation- usually hemorrhagic. Involvement of 
underlying muscle is always more extensive than the evident skin involvement. The blister serosanguinous fluid will contain GPR, but inflammatory cells are infrequent [4]. Pathology: there is cell lysis and gas formation in connective tissue and muscle with minimal inflammation [4].

The diagnosis of clostridial myonecrosis requires a high index of suspicion, since the infection spreads rapidly and death may occur within 24-48 h. Gram stain of a bulla allows for a timely diagnosis. Anaerobic cultures should be sent to determine appropriate antibiotic use. Imaging with X-ray or computed tomography (CT) scan can demonstrate soft tissue gas (a late finding) and help determine the extent of infection [3].

(b) Spreading cellulitis with clostridial septicemia is a fulminant infection in neutropenic patients. A small area of purplish discoloration develops, often on the flank or abdominal wall. The lesion will expand rapidly over several hours, and more lesions appear in other areas. As it progresses, the lesions turn into brownish to blackish color with blister formation and crepitation. Clostridium can be captured in the fluid, but without inflammatory cells [4].

Clostridium septicum has been associated with necrotizing enterocolitis (typhlitis), which typically is a fulminant infection of the intestines in neutropenic patients [18].

Corynebacterium jeikeium (C. jeikeium) Corynebacterium species are part of the normal skin flora. The most common sites of colonization by $\mathrm{C}$. jeikeium are the perineum, rectal, inguinal and axillary areas. Corynebacteria species rarely cause infection. Infections due to C. jeikeium are mainly seen in immunosuppressed patients, especially those with neutropenia and indwelling catheters [19].

Primary cutaneous infection with C. jeikeium occurs at breaks in the skin barrier due to bone marrow biopsy, intravascular catheter insertion, groin or perirectal areas (anal fissures) which serve as a portal of entry into the bloodstream, leading to septicemia. Primary skin lesions typically present as cellulitis or wound infection [20].

Secondary skin and soft tissue infections with C. jeikeium develop in almost $30-50 \%$ of neutropenic patient with $\mathrm{C}$ jeikeium bacteremia [4]. It may present as single to multiple, nontender, noninflamed $2 \times 2 \mathrm{~cm}$ subcutaneous nodules that do not spontaneously drain but are purulent upon surgical drainage [19]; bright red, non-blanching papules with satellite petechiae and central necrosis or pustulation on the trunk and/or extremities [20].

Staphylococci species are frequent colonizers of normal skin but commonly infect immunocompromised individuals. Leukopenic patients are at greatest risk for infection. Common infections include impetigo, furuncles, carbuncles, ecthyma, folliculitis, cellulitis etc. In immunocompromised patient, vesicle or bullous eruption can be seen [3].

Streptococcal infections in bone marrow transplant (BMT) recipients and acute leukemia patients are serious and may be clinically atypical [4]. Group A or Group B streptococcus cutaneous manifestations include erysipelas/cellulitis in only $25 \%$, the rest of the cases are infections of wounds or ulcers, myositis, necrotizing fasciitis, and toxic shock syndrome. 
Viridans group streptococci (VGS) are part of the normal microbial flora of humans, risk factors for invasive disease is mucositis and neutropenia.

A case control study of VGS sepsis was done at the University of Texas M. D. Anderson Cancer Center in Houston, where controls were randomly selected from patients with other Gram-positive septicemia. Flushing of the face and a rash occurred in nearly $60 \%$ of these patients but were uncommon in the control group. The rash was usually erythematous maculopapular, beginning on the trunk and progressing to the face and extremities compatible with toxic shock syndrome picture. In $25 \%$ of the cases, the rash resulted in desquamation of the palms and soles 1-2 weeks later. Ten percent of the patients developed ARDS, shock, or renal failure and died despite more than 4 days of vancomycin therapy. The patients with VSG had a higher rate of oral mucositis, BMT, and severe neutropenia $<100$, on antacid or $\mathrm{H} 2$ antagonist therapy, and parenteral nutrition [4, 21].

\section{Mycobacteria}

Mycobacterial cutaneous infection occurs as a result of direct inoculation from an exogenous source, or through contiguous or hematogenous spread. Cutaneous mycobacteria can exhibit a large spectrum of morphological presentation. Infection may be caused by Mycobacterium tuberculosis (MTb), Mycobacterium avium intracellulare complex (MAC), and other non-tuberculous mycobacteria (NTM) including rapidly growing mycobacteria (RGM), although $\mathrm{MTb}$ is commonly seen in HIV patients those undergoing solid organ transplant.

Tuberculosis in neutropenic patients is one of the most serious opportunistic infections encountered. Cutaneous miliary tuberculosis presents as erythematous to brown papules, which can become vesicular or pustular. The tiny vesicles or pustules rupture and form a central crust on the papule. Removal of the crust leaves a minute but sharply defined umbilication [3].

Pathology results are characterized by the absence of a granulomatous response, giant cells, and true caseating granulomas [6]. An acid-fast stain (if the diagnosis is considered and the stain requested) usually shows numerous acid-fast bacilli (AFB) seen quite easily [3].

NTM are ubiquitous in the environment and reside in soil and water. Of the over 150 NTM species, only 25 are known to cause disease in humans [22]. Skin and soft tissue infections are most commonly caused by Mycobacterium marinum and Mycobacterium ulcerans, which are both slowly growing mycobacteria [23].

In the immunocompromised, the typical history of previous trauma or surgery is absent, thus cutaneous Mycobacteria infection are most probably the result of hematogenic dissemination, resulting in multiple skin lesions. This clinical entity is well described in patients with $\mathrm{T}$ cell mediated immunodeficiency such as AIDS, solid organ and bone marrow transplant patients, but infrequently in patients with hematological malignancies, either with or without neutropenia [24]. 
Case studies described highlight the variety of immune system dysfunction (T-cell, humoral, or granulocyte-related) which combined may have predisposed to the disseminated NTM in neutropenic patients [13, 25]. Chronic indwelling central venous catheter-related infection is one of the most common risk factor for disseminated nontuberculous mycobacterial infection [26].

The lesions are usually more extensive in the immunosuppressed population, nonspecific and heterogeneous. They range from panniculitis (Fig. 2), single to widespread nodules, sinus tracts, nonhealing ulcers, subcutaneous abscesses, or erythematous plaques (Fig. 3) [3].

Mycobacterium malmoense, a slow-growing mycobacteria may cause cutaneous lesions that were described in a 75 year old neutropenic man with myelodysplastic syndrome, who was also receiving corticosteroids. He developed papulokeratotic pinkish nodules lesions in a sporotrichoid distribution on the arm that later ulcerated and some became necrotic [27].

Mycobacterium avium-intracellulare complex (MAC), is a common opportunistic pathogen in AIDS patients and patients with cell medicated immunodeficiency mainly, it can also cause infections in neutropenic patients. The clinical manifestations include widespread erythematous tender nodules on the upper, and lower extremities, chest and abdomen that can ulcerate (Fig. 2). The infection is usually associated with bacteremia and cavitary lung lesions may also be present. Skin biopsy demonstrates areas of dermal and subcutaneous necrosis with numerous acid-fast bacilli [3].

Mycobacterium fortuitum, Mycobacterium abscessus, and Mycobacterium chelonae are collectively referred to as rapidly growing mycobacteria (RGM). These environmental pathogens may cause skin infections that usually occur following trauma or surgical procedures and injections, which is usually not present in immunocompromised patients. M. fortuitum infection is more common in immunocompetent patients, while $M$. chelonae and $M$ abscessus more often infects immunocompromised patients [3].

Fig. 2 Indurated tender red lesions on the extremities of an immunocompromised patient - skin biopsy was consistent with infectious panniculitis secondary to Mycobacterium chelonae

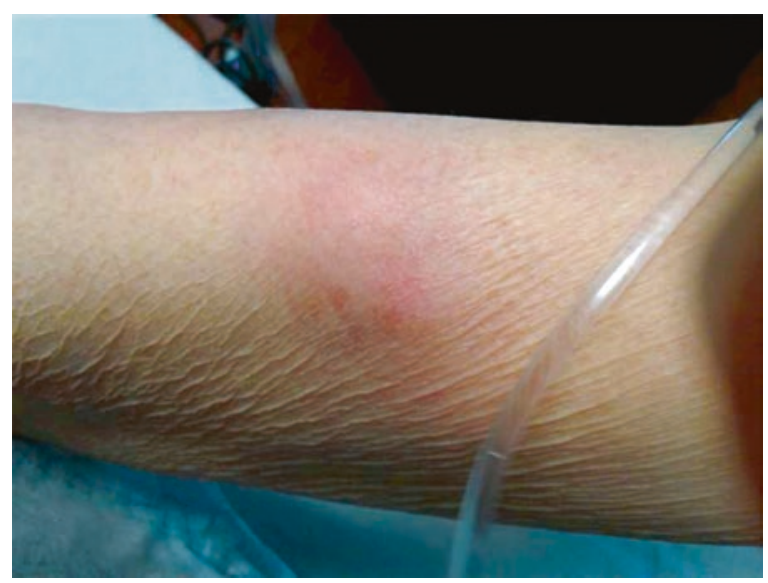



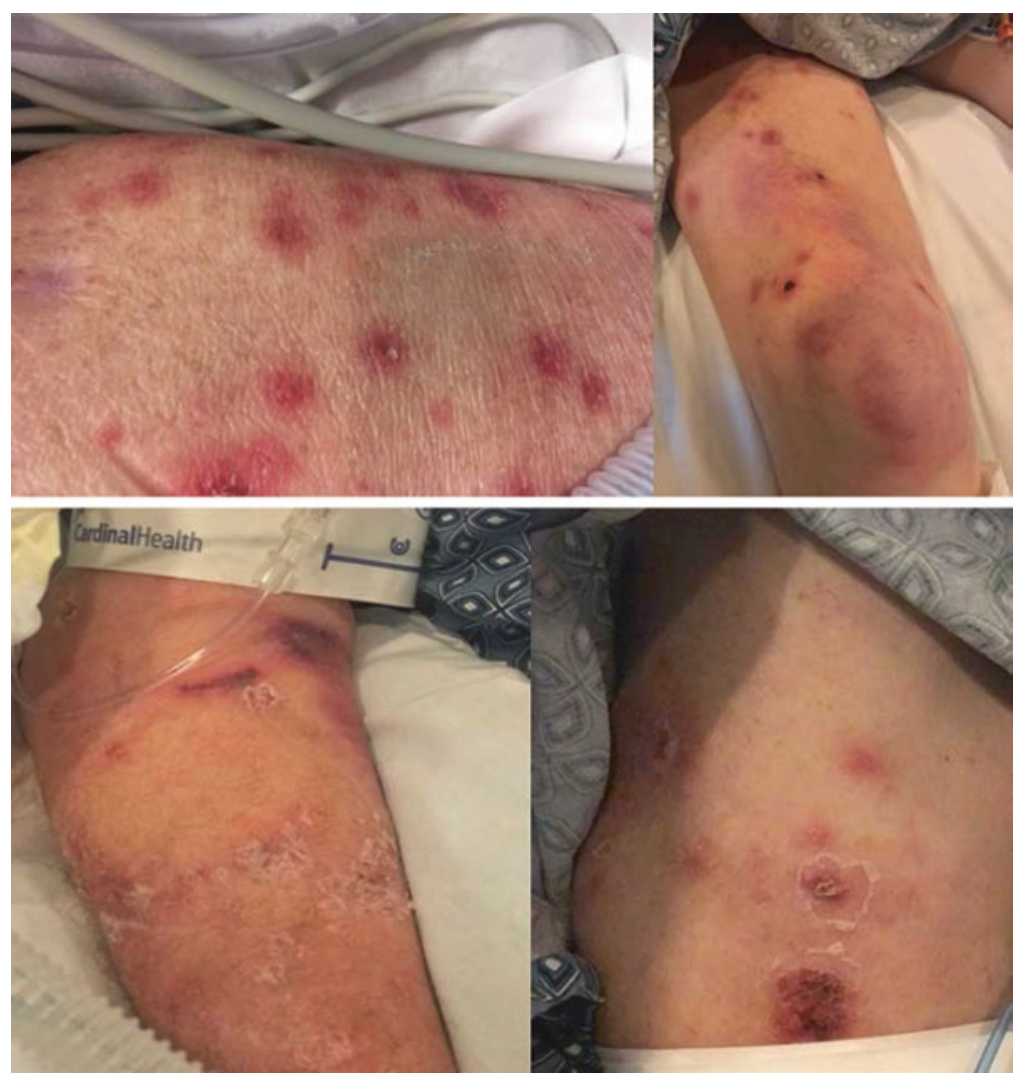

Fig. 3 Disseminated mycobacterium avium intracellulare complex in neutropenic patients

Disseminated cutaneous Mycobacterium chelonae infection in neutropenic patients can cause neutropenic fever and multiple dusky red to purple, tender, subcutaneous nodules on the face, arms, and legs, resulting in firm purple ulcerating nodules. Within weeks, the nodules can developed larger plaques that can ulcerate [25].

It is essential to maintain a high index of suspicion for atypical mycobacterial infection, as acid-fast organisms may be difficult to identify on histologic sections, and many of these fastidious pathogens are challenging to grow in culture, often requiring weeks for the culture to turn positive, and in some cases requiring specific media. Polymerase chain reaction (PCR) is an emerging technique, which may aid in the diagnosis of mycobacterial infections. Speciation of the offending organism is crucial, as the antibiotic susceptibility profiles differ among the organisms and are essential to achieving therapeutic success [3]. 


\section{Viral Infections}

Neutrophils are not the primary defense against viral infections. However hematologic malignancy is the most prevalent background of neutropenic patients with cutaneous lesions $95.3 \%$ based on a retrospective review from 2009-2014 [28]. In this population, the combination of dysfuction of different parts of the immune system provides sufficient opportunity for viruses such as herpesiviridae to reactivate and cause disseminated infection [13].

\section{Herpes Simplex Virus}

Infections caused by herpes simplex virus (HSV) are exceedingly common inpatient with acute leukemia and post bone marrow transplant [4].

They are divided into primary and secondary (recurrent) forms, which are selflimited in normal hosts. In the majority of immunocompromised hosts, the HSV infection is not primary but rather reactivation of latent HSV.

Most herpetic infections involve the oral mucosa, lips, and nares. Genital lesions are less common [4]. Any periorificial ulceration in the immunocompromised host should be considered herpes simplex until proven otherwise.

Symptoms such as paresthesia, burning sensation or pruritus precede the lesions. They begin as vesicles that rupture spontaneously, leaving ulcerations that enlarge, coalesce and become encrusted. Infection often follows a chronic course in immunosuppressed patients, resulting in larger, deeper, painful necrotic erosive lesions known as phagedena. Superinfection by bacteria or fungi, esp. by Staphylococcus aureus may occur, which may distort the initial appearance, resulting in misdiagnosis. Healing typically occurs within 1 week in normal hosts, but may take up to 5 weeks in immunocompromised patients $[3,4]$.

Intraoral HSV can present as ulcerations with polycyclic borders, linear fissures on the tongue known as herpetic geometric glossitis. The break of mucosal integrity provides a portal of invasion by both pathogenic and normal microbial flora inhabiting the mouth. Other variant of atypical herpes simplex virus infection is deep linear fissures in the skin folds (inframammary, infra-abdominal, inguinal, or vulvar) termed the "knife-cut sign", and bilateral mutidigit herpetic whitlow (Fig. 4) reported in a patient after receiving chemotherapy for chronic lymphocytic leukemia (CLL) [3].

Both oral and intravenous acyclovir has been effective in preventing HSV infection in patients with acute leukemia and BMT recipients. 
Fig. 4 Herpetic whitlow

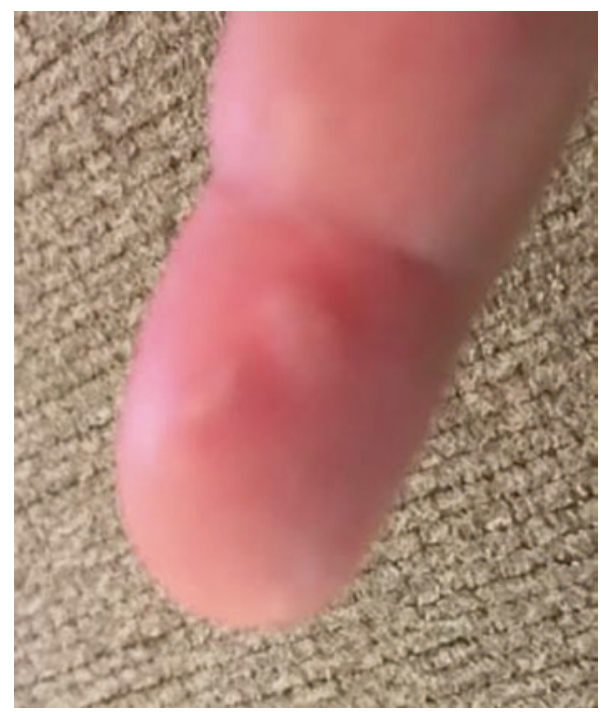

\section{Herpes Zoster Virus}

Herpes zoster (HZ) represents reactivation of the varicella-zoster (VZ) virus. Following the primary varicella infection (chicken pox), the virus remains dormant in a dorsal root ganglion or a cranial nerve ganglion. Reactivation occurs as herpes zoster with cutaneous vesicles and dermatomal pain. The skin lesions of VZ in the immunocompromised host occur in three forms: (a) dermatomal HZ (which may be less than 3 contiguous dermatomes), (b) disseminated $\mathrm{HZ}$ and (c) chronic $\mathrm{HZ}$ or recurrent $\mathrm{HZ}$. In patients with malignant disease, the incidence of $\mathrm{HZ}$ is further increased: highest in lymphoreticular disorders, Hodgkin's disease, then nonHodgkin's lymphomas, followed by solid tumors, particularly small cell carcinoma of the lung [29] (Fig. 5).

\section{Cytomegalovirus (CMV)}

CMV (HHV-5) is a common human viral infection affecting $40-100 \%$ of adults worldwide. Acute infections are often asymptomatic, but once the infection is acquired, there is a lifelong latency along with the risk of intermittent reactivation. CMV disease is due to reactivation of latent virus following iatrogenic immunosuppression in organ transplantation, and cancer chemotherapy. Skin manifestations of CMV are rare in any setting, and very nonspecific (exanthematous, maculopapular, or morbilliform eruptions) and therefore diagnosis is often delayed. The cardinal manifestation of CMV infection in the skin is a chronic painful ulcer of the anal, perianal, or anogenital area. Although uncommon, oral manifestations of CMV 

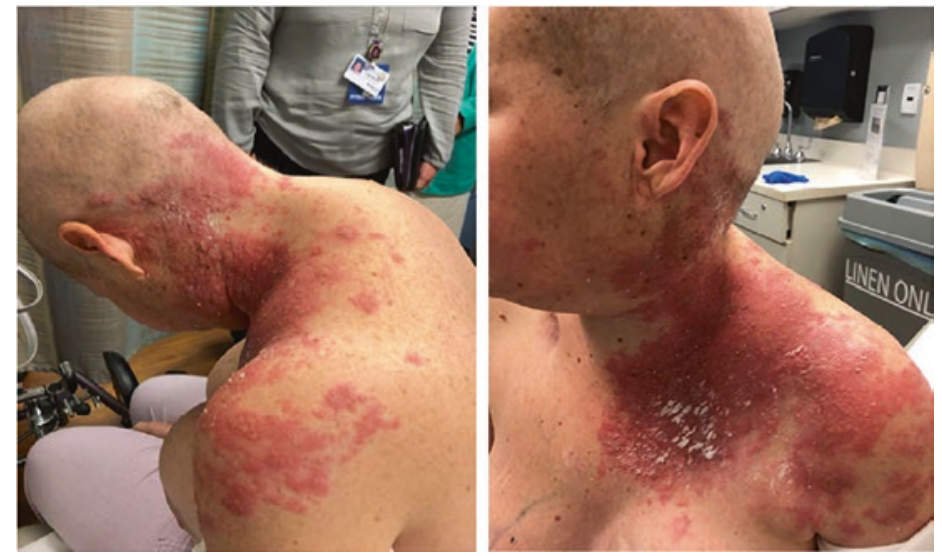

Fig. 5 Disseminated zoster in a neutropenic patient with acute myelogeneous leukemia

infection have included painful erosions or ulcers of the tongue, buccal mucosa, and pharynx. Skin biopsy will confirm the diagnosis by demonstrating the characteristic large intranuclear inclusions with a surrounding halo, or "owl's eye" characteristic of CMV. The diagnosis is confirmed with immunoperoxidase stain [3].

\section{Fungal Infections}

There are many fungi including yeast and mold, which are of medical importance, particularly in the immunocompromised population. Patients with hematologic malignancies and those receiving chemotherapy are at highest risk [30]. Fungal infections have many clinical presentations including dermatologic manifestations. These infections can be localized after local trauma, progress to invasive disease or dissemination, or result from hematogenous infection.

\section{Candida}

Superficial cutaneous Candida infections such as intertrigo and vaginitis present similarly to immunocompetent patients [30]. However, they may be more common in patients who require systemic antibiotics or steroids during their oncologic treatment. Most superficial infections can be treated with topical anti-fungal [31].

Disseminated candidiasis can occur in neutropenic and non-neutropenic populations. The portal of entry is typically via gastrointestinal translocation or introduction from a central line. In patients with hematologic malignancies and stem-cell transplantation, non-albicans species predominate including C. glabrata, C. krusei and $C$. tropicalis [32-36]. In solid tumors, approximately half of disseminated can- 
Fig. 6 Scattered papules of the back in a patient with disseminated Candida tropicalis in the setting of acute myelogenous leukemia and neutropenia

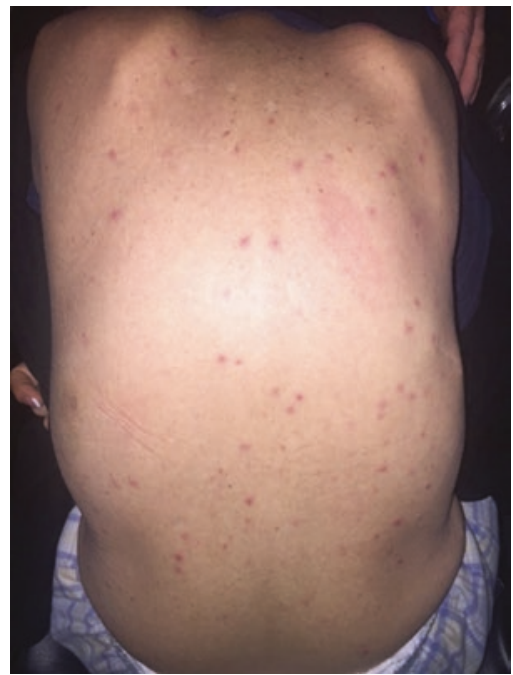

didiasis is secondary to $C$. albicans [32]. The epidemiology of these infections is likely related to the choice of fungal prophylaxis and resistance patterns of nonalbicans species [34]. The frequency of cutaneous manifestations in the setting of candidemia is reported to occur in $11-44 \%$ of cases [34]. The typical skin lesions are 5-10 mm pink papules, which rarely lead to eschar formation or skin necrosis. There are often numerous papules involving the trunk and proximal extremities [30]. Some lesions may be purpuric, especially if thrombocytopenia is also present [34]. Mortality associated with candidemia has been reported to be over $50 \%$, therefore prompt recognition of skin lesions and initiation of empiric treatment is important [35]. Diagnostics can be challenging, positive blood cultures occur in less than $50 \%$ of the cases. It is important to consider obtaining tissue for histopathology and culture or using novel non-culture diagnostics. Histology typically shows fungal elements including pseudohyphae, hyphae and yeast within the dermis or blood vessels [34]. An echinocandin such as micafungin is recommended as empiric treatment in most patients [31] (Fig. 6).

\section{Fusarium}

Skin manifestations occur in up to $70 \%$ of patients with Fusariosis and can present as a localized or disseminated infection [36]. Neutropenic patients with hematologic malignancies are at high risk for these mold infections [37]. An important clinical presentation of localized fusarium infection in the neutropenic population is fungal paronychia, which presents as erythema and swelling of the periungal skin [30]. Fungal paronychia should be suspected in neutropenic patients who have underlying onychomycosis, develop an eschar, and do not improve on systemic antibiotics [30]. Infection may be preceded by minor trauma [38]. Local infections 
can lead to fungemia and disseminated infection. Disseminated Fusarium infection typically presents as multiple erythematous papules or nodules, which can develop central necrosis or targetoid appearance. They often present at different stages of evolution and are widely distributed on the trunk and extremities [36].

Skin biopsy with histopathology and culture are helpful in securing a diagnosis. Histology will show ballooning branching septated hyphae, which can invade capillaries and blood vessels [39]. Skin lesions may appear before systemic symptoms develop or blood cultures become positive [30]. Unlike other disseminated mold infections, blood cultures are often positive in disseminated Fusariosis and should be collected when Fusarium is suspected [37]. Empiric treatment with liposomal amphotericin B is recommended [40]. More details about therapy please refer to the chapter titled fungal infections (Figs. 7, 8, and 9).

\section{Aspergillus}

Although aspergillus is one of the most common etiologies of invasive fungal infections in patients with underlying malignancies, dermatologic manifestations occurs in less than $5 \%$ of cases $[41,42]$. Cutaneous involvement can present as a localized infection by direct skin inoculation or via hematogenous dissemination. Blood cultures are typically negative in disseminated infection therefore it is recommended to obtain tissue biopsy with culture and histopathology. Skin manifestations are similar to Fusarium infections. Primary or localized lesions can present as cellulitis with focal erythema and swelling that can progress leading to the formation of bullae, necrotic ulcers and eschars. Invasive fungal sinusitis secondary to Aspergillus sp. can also extend to the skin leading to facial cellulitis; this is covered in further detail in the chapter about fungal infections. Disseminated infection typically presents as scattered erythematous papules or nodules $[30,41]$. Histology will show septated narrow hyphae with $45^{\circ}$ branching and club-shaped pseudohyphae [43]. Treatment with

Fig. 7 Paronychia and cellulitis secondary to Fusarium sp. in a patient with acute myelogenous leukemia and prolonged neutropenia

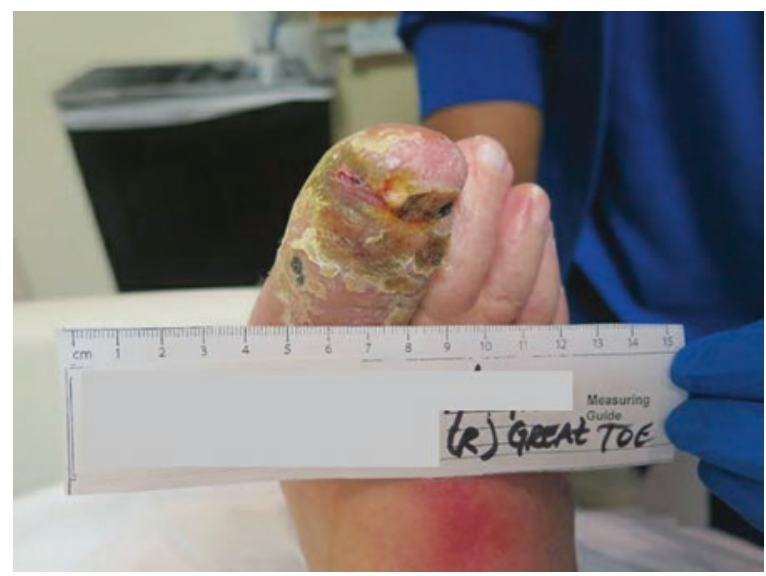


Fig. 8 Erythematous nodules with surrounding erythema in a patient with acute myelogenous leukemia and disseminated Fusarium

Fig. 9 Necrotic eschar with surrounding petechial erythema secondary to localized Fusarium infection in a patient with underlying B-cell lymphoma

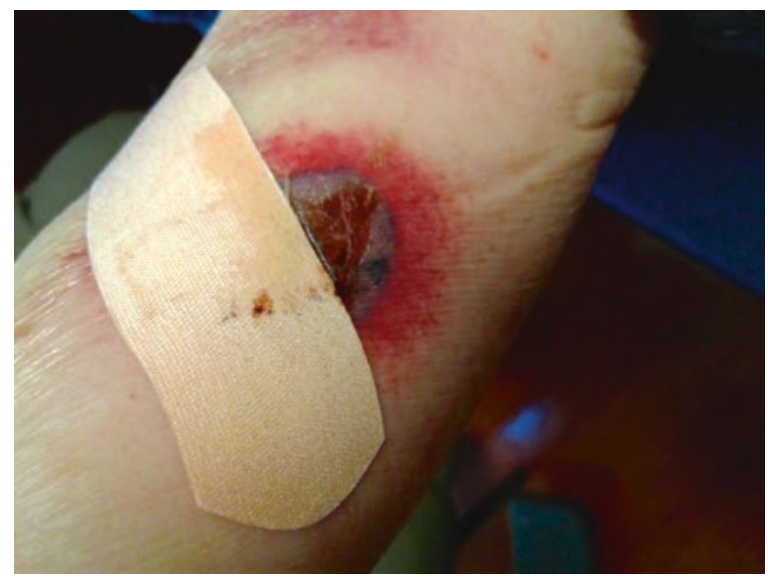

triazoles such as voriconazole is the preferred anti-fungal unless there is a contraindication to this class and then liposomal amphotericin B would be recommended [44].

\section{Mucormycosis}

The most common Zygomycetes to cause clinical disease are Rhizopus, Mucor, Rhizomucor, Lichtheimia, and Cunninghamella spp. [45]. In patients with underlying malignancy, the primary site of mucormycosis is cutaneous in $12 \%$ of cases 
[46]. Unlike other fungal infections, cutaneous mucormycosis most often occurs by direct inoculation rather than dissemination. Skin lesions may begin as a small erythematous macule but typically develop a black necrotic eschar with surrounding erythema and swelling. If there is concern for disseminated disease it is important to look for metastatic skin lesions [47]. Patients with rhinocerebral mucormycosis may present with orbital or facial cellulitis with a classic black necrotic eschar [45]. Obtaining tissue histopathology with culture is essential for diagnosis. Histologic examination will show broad non septate hyphae branching at $90^{\circ}$ [43]. There may be vascular invasion leading to thrombosis and infarction [47]. Liposomal amphotericin B is treatment of choice [48].

\section{Other Fungi}

There are several other fungal infections that are less common but also important to consider in the evaluation of a cancer patient with a rash. Geographic location and previous travel can increase risk for endemic fungal infections.

Histoplasmosis is endemic in the Ohio River valley and parts of Central America. Cutaneous lesions typically present in cases of disseminated disease and often mimic other dermatologic infections due its' ability to manifest with many skin findings including papules, plaques, nodules, ulcers or pustules. Histopathology typically shows granulomas with lymphohistiocytic infiltrates and yeast within or outside macrophages [49].

Blastomycosis can be found in the Ohio and Mississippi river valleys and in the southeastern United States [50]. Skin lesions begin as an erythematous papule and evolve into a scaling or vegetative plaque. Histopathology may show pseudoepitheliomatous hyperplasia with neutrophilic abscesses or noncaseating granulomas. Tissue culture will reveal a thick-walled yeast with broad-based budding [51, 52].

Coccidiomycosis is found in the southwestern United States and can present with cutaneous findings when dissemination or reactivation occurs. Like histoplasmosis, disseminated coccidomycosis can present in many cutaneous forms including papules, plaques, nodules and ulcers. Histopathologic examination can show granulomatous or suppurative inflammation with numerous eosinophils. Coccidiomycosis can also result in reactive skin eruptions such as erythema nodosum, sweet's syndrome, interstitial granulomatous dermatitis, and exanthems. These presentations typically occur in the setting of pulmonary coccidiomycosis. Histopathology for reactive eruptions is variable depending on the skin manifestation and will have sterile tissue culture [53].

Cryptococcus spp. are encapsulated yeast, which are ubiquitous in the environment, often being found in the soil and pigeon droppings. These infections are commonly reported in the HIV population however they can occur in other immunocompromising conditions including malignancies. Like many other cutaneous fungal infections, skin lesions are polymorphous. Cutaneous findings can represent primary infection but most often occur in the setting of disseminated disease. 
Therefore it is important to look for other organ involvement when cutaneous cryptococcal infection is diagnosed [54]. Obtaining histopathology with tissue culture is essential for diagnosis, using stains such as mucicarmine and alcian blue to identify the capsule [55].

\section{Non Infectious Differential}

Although it is always important to consider infectious dermatologic etiologies in cancer patients who present with a rash, there are also several non-infectious skin diseases that are important to recognize. Cancers themselves can cause unique skin pathology however there are also paraneoplastic and inflammatory skin disorders that can occur secondary to malignancies. Obtaining a thorough history and review of medications can assist with determining the diagnosis but skin biopsy is usually necessary given many diseases have similar findings on clinical examination.

\section{Leukemia cutis}

Leukemia cutis (LC) is an extra-medullary presentation of leukemia which is due to the invasion of malignant cells into the skin [56]. LC is most commonly associated with AML but can also occur with other underlying myeloid disorders and rarely presents without bone marrow involvement. Typical skin findings are erythematous or violaceous papules, nodules or plaques. The lesions have also been described as rubbery or shiny and may present with bullae $[57,58]$. They can be pruritic but are usually not painful. Skin biopsy with immunohistochemical analysis is essential for diagnosis [59]. Histopathology will show leukemic cells, typically myeloblasts, invading the dermis. Prognosis is typically poor and may represent progression of underlying malignancy [58] (Fig. 10).

Fig. 10 Skin examination shows a tense bullae with surrounding erythema. Histopathology revealed leukemia cutis in the setting of AML

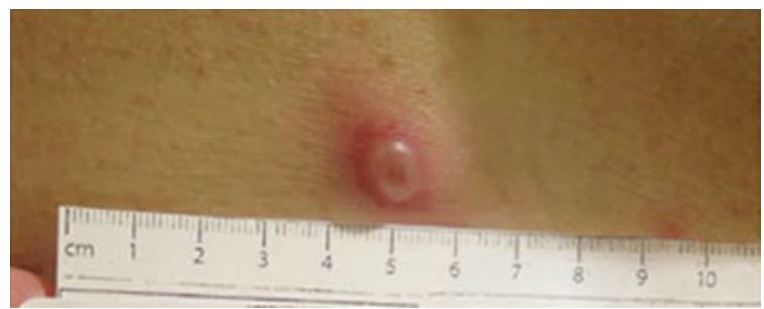




\section{Sweet's syndrome (Acute Febrile Neutrophilic Dermatosis)}

Sweet's syndrome (SS) is characterized by the development of fever, a painful rash, and leukocytosis [58]. SS can occur in the presence of an underlying malignancy such as AML, CML, MDS or myelofibrosis. It is also known to be drug-induced and can occur secondary to infections, classically it is described after an upper respiratory tract infection [60]. The skin lesions are often erythematous or violaceous papules, nodules or plaques which are tender. Vesicles or bullae can develop due to edema of the upper dermis [60]. A unique finding in SS is pathergy. Cutaneous findings may appear after trauma including venipuncture or biopsy [61]. Histopathology shows neutrophilic infiltrates of the dermal papillae without evidence of leukoclastic vasculitis. Unlike leukemia cutis, the neutrophils that infiltrate the dermis are typically mature $[60,62]$. When considering SS it is important to rule out an underlying infection; an important differential diagnosis is pyoderma gangrenosum. Treatment of the underlying malignancy is necessary but steroids can also be utilized [63] (Fig. 11).

\section{Pyoderma Gangrenosum}

Pyoderma gangrenosum is an ulcerative neutrophilic dermatosis. PG is associated with many inflammatory and autoimmune conditions including both hematologic malignancies and solid tumors [64]. It is typically a diagnosis of exclusion but is characterized by a rapidly developing, painful ulceration with undermined borders. Like Sweet's Syndrome it may exhibit pathergy [65]. The base of the ulcer is typically necrotic with a surrounding erythematous or violaceous halo [66]. The bullous subtype is characterized by bullae formation preceding the ulceration and is commonly associated with hematologic malignancies [58]. Skin biopsy from the border

Fig. 11 Erythematous and edematous plaque of the hand with large bullae of the forearm. There are several annular erythematous lesions of the proximal arm. The two round hyperpigmented lesions of the dorsum of the hand represent punch biopsy sites.

Histopathology revealed sweet's syndrome in the setting of MDS

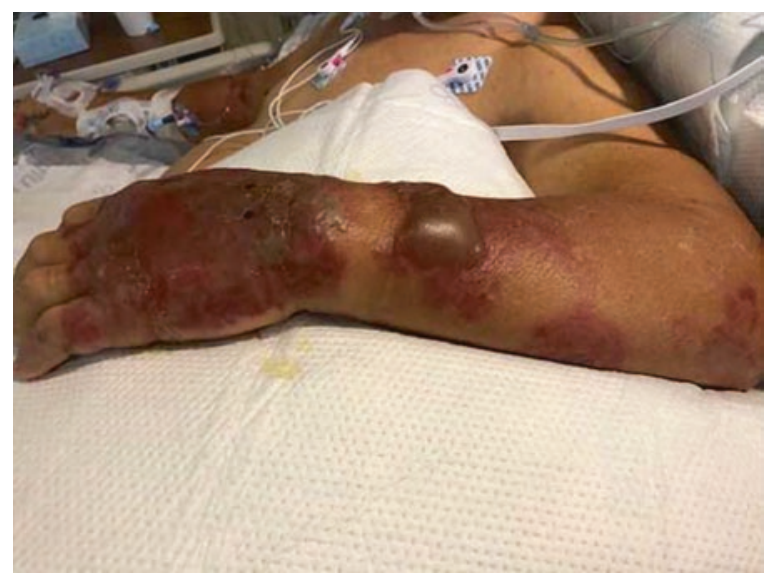


of the ulcer should be obtained although it is difficult to diagnose PG based on pathology due to changing histology with the evolution of the ulcer. Examination may reveal dense neutrophilic infiltrates with leukoclastic vasculitis or necrosis [67]. Treatment with steroids usually results in rapid improvement [66].

\section{Vasculitis}

Paraneoplastic vasculitis is most commonly associated with MDS and other hematologic malignancies [68]. It is characterized by non-blanchable palpable purpura which may be painful or pruritic [58, 69]. Biopsy is essential for diagnosis and will show necrotizing leukocytoclastic vasculitis with fibrinoid necrosis and neutrophil infiltration of the vessel wall [70]. The presentation of vasculitis may precede the diagnosis of cancer but can also present at all stages of disease [68]. Treatment of the underlying malignancy can result in resolution of the vasculitis [58].

\section{Medication Reactions}

Many medications that patients have exposure to during the treatment of malignancies can lead to rashes and skin pathology. It is important to monitor for allergic drug rashes which usually resolve with discontinuation of the inciting medication. StevenJohnson syndrome (SJS) and toxic epidermal necrolysis (TEN) are both drug reactions that have a high mortality rate due to epidermal sloughing. SJS and TEN may begin with a prodrome which includes high fever. Skin findings may begin with erythematous macules or targetoid lesions progressing to the development of bullae and erosions [71]. Nikolsky sign is often positive. Many patients will also have mucosal involvement [72]. The two conditions are distinguished from each other based on body surface involvement; SJS involves $<10 \%$ and TEN involves $>30 \%$ with an overlap of disease characterization between 10-30\% [71]. Many drugs have been linked to the development of SJS or TEN, particularly antibiotics. Sulfonamide antibiotics are most commonly associated with SJS or TEN however all antibiotics should be considered as an etiology [71,73] (Figs. 12 and 13).

Toxic erythema of chemotherapy is a nonallergic reaction that is caused by many chemotherapeutic agents. Toxic erythema of chemotherapy presents with painful erythema and edema which can be present on the hands and feet or intertriginous areas. It is most commonly associated with methotrexate, cytarabine, anthracyclines, 5-fluorouracil, and taxanes [58]. Multikinase inhibitors such as sorafenib can cause a hand-foot-skin reaction which is characterized by painful hyperkeratotic plaques which develop in areas of friction such as the fingertips and joints [58, 74]. Tyrosine kinase inhibitors including imatinib or dasatinib are also commonly associated with cutaneous reactions which can include a maculopapular rash and facial edema $[58,75]$. 
Fig. 12 Cutaneous examination showing erythematous macules with areas of central ulceration consistent with StevensJohnson Syndrome in a patient with a history of Burkitt's Lymphoma receiving vancomycin

Fig. 13 The above patient in Fig. 12 developed numerous bullae with areas of erosions and sloughing leading to denuded skin consistent with the diagnosis of StevensJohnson Syndrome
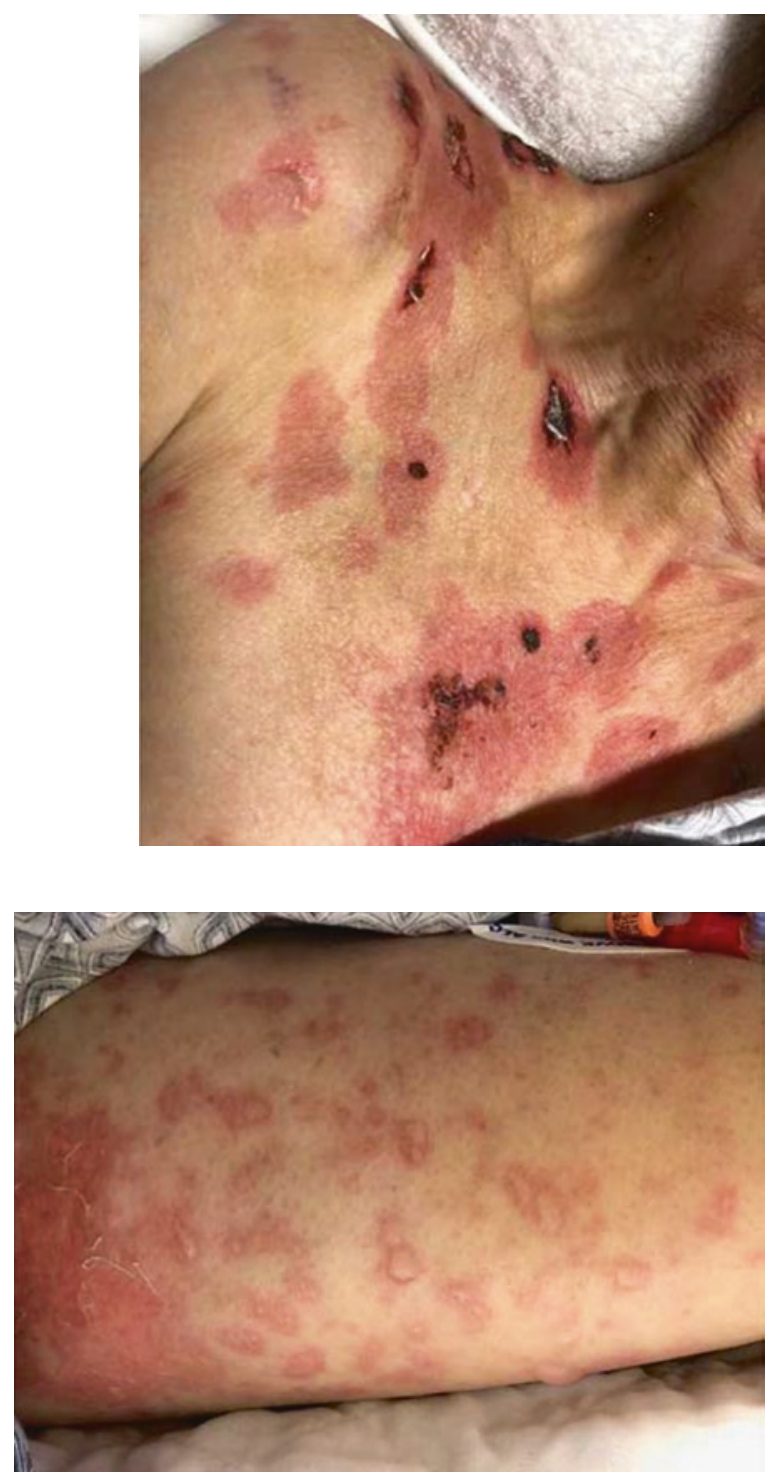

\section{Key Points}

\begin{tabular}{l|l}
\hline Rash description & Pathogen \\
\hline Cellulitis & Staphylococcus, Streptococcus including Streptococcus Pneumonia, \\
& Bacillus, Clostridium, Corynebacterium JK, Pseudomonas, E.coli, Serratia, \\
& Stenotrophomonas, Aeromonas, Chromobacterium. \\
& Aspergillus, Fusarium, Candidiasis, Mucormycosis. \\
& Non Tuberculous Mycobacteria. \\
\hline
\end{tabular}




\begin{tabular}{l|l}
\hline Rash description & Pathogen \\
\hline Pustules & $\begin{array}{l}\text { Staphylococcus aureus, Bacillus, Corynebacterium, Gram negative bacteria } \\
\text { (mainly Ecoli, Citrobacter, chromobacterium). } \\
\text { Mycobacteria. } \\
\text { Candidiasis, Histoplasmosis, Aspergillosis, Fusarium. } \\
\text { HSV/HZV. }\end{array}$ \\
\hline Necrotic Lesion & $\begin{array}{l}\text { Pseudomonas, other Gram negative bacilli (mainly E coli, } \\
\text { Stenotrophomonas, Citrobacter), Bacillus, Clostridium, Corynebacterium. } \\
\text { Aspergillus, Candidiasis, Mucormycosis, Fusarium. } \\
\text { HSV/HZV/CMV. }\end{array}$ \\
\hline nodules & $\begin{array}{l}\text { Staphylococcus aureus, Corynebacterium JK, Pseudomonas, E coli, } \\
\text { Stenotrophomonas. } \\
\text { Mycobacteria. }\end{array}$ \\
\hline Aspergillosis, Fusarium. Candidiasis, Coccidiomycosis, Cryptococcus, \\
Histoplasmosis.
\end{tabular}

\section{References}

1. Kryeziu E, Kryeziu K, Bajraktari G, Abazi M, Zylfiu B, Rudhani I, Sh S, Ukimeri A, Brovina A, Sh D, Telaku S. Ecthyma gangrenosum in a patient with acute leukemia. Med Arh. 2010;64(6):373-4.

2. Brady GP, Bolivar R, Fainstein V, et al. Infections caused by Pseudomonas aeruginosa. Rev Infect Dis. 1983;5:279-313.

3. Grossman ME, Fox LP, Kovarik C, Rosenbach M. Cutaneous manifestations of infection in the immunocompromised host. 2nd ed. New York: Springer Science+Business Media, LLC; 2012. https://doi.org/10.1007/978-1-4419-1578-8.

4. Bodey GP. Dermatologic manifestations of infections in neutropenic patients. Infect Dis Clin N Am. 1994;8(3):655-75. Review. 
5. Mull JD, Callahan WS. The role of the elastase of Pseudomonas aeruginosa in experimental infection. Exp Mol Pathol. 1995;4:567-75.

6. Brzozowski D, Ross DC. Upper limb Escherichia coli cellulitis in the immunocompromised. J Hand Surg Br. 1997;22(5):679-80.

7. Livingston W, Grossman ME, Garvey G. Hemorrhagic bullae in association with Enterobacter cloacae septicemia. J Am Acad Dermatol. 1992;27:637-8.

8. Chapman RA, Van Slyck EJ, Madhavan T. Skin lesions associated with E. coli sepsis in a patient with acute leukemia. Henry Ford Hosp Med J. 1980;28(1):47-8.

9. Grant MD, Horowitz HI, Lorian V. Gangrenous ulcer and septicemia due to citrobacter. N Engl J Med. 1969;280(23):1286-7.

10. Gössl M, Eggebrecht H. Necrotizing skin ulceration in antibiotic-induced agranulocytosis. Mayo Clin Proc. 2006;81(12):1527.

11. Sakhnini E, Weissmann A, Oren I. Fulminant Stenotrophomonas maltophilia soft tissue infection in immunocompromised patients: an outbreak transmitted via tap water. Am J Med Sci. 2002;323(5):269-72.

12. Harris RL, Faintein V, Elting L, et al. Bacteremia caused by Aeromonas species hospitalized cancer patients. Rev Infect Dis. 1985;7:314-20.

13. Klastersky J. Infections in immunocompromised patients. I. Pathogenesis, etiology, and diagnosis. Clin Ther. 1985;8(1):90-9.

14. Cone LA, Dreisbach L, Potts BE, Comess BE, Burleigh WA. Fatal Bacillus cereus endocarditis masquerading as an anthrax-like infection in a patient with acute lymphoblastic leukemia: case report. J Heart Valve Dis. 2005;14(1):37-9.

15. Mori T, Tokuhira M, Takae Y, et al. Successful non-surgical treatment of brain abscess and necrotizing fasciitis caused by Bacillus cereus. Intern Med. 2002;41(8):671-3.

16. Groschel D, Burgess MA, Bodey GP. Gas gangrene-like infection with Bacillus cereus in a lymphoma patient. Cancer. 1976;37:988-91.

17. Bodey GP, Rodriguez S, Fainstein V, et al. Clostridial bacteremia in cancer patients. Cancer. 1991;67:1928-42.

18. Hopkins DG, Kushner JP. Clostridial species in the pathogenesis of necrotizing enterocolitis in patients with neutropenia. Am J Hematol. 1983;14:289-95.

19. Van der Lelie H, Leverstein-Van Hall M, Mertens M, et al. Corynebacterium CDC group JK (Corynebacterium jeikeium) sepsis in haematological patients: a report of three cases and a systematic literature review. Scand J Infect Dis. 1995;27(6):581-4.

20. Olson JM, Nguyen VQ, Yoo J, Kuechle MK. Cutaneous manifestations of Corynebacterium jeikeium sepsis. Int J Dermatol. 2009;48(8):886-8.

21. Elting LS, Bodey GP, Keefe BH. Septicemia and shock syndrome due to viridans streptococci: a case - control study of predisposing factors. Clin Infect Dis. 1992;14:1201-7.

22. Van Ingen J. Diagnosis of nontuberculous mycobacterial infections. Semin Respir Crit Care Med. 2013;34(pg):103-9.

23. Roukens AH, Mendels EJ, Verbeet NL, Borne PA v d, Nicolae-Cristea AR, Bentvelsen RG, van Doorn R, de Boer MG. Disseminated cutaneous Mycobacterium chelonae infection in a patient with acute myeloid leukemia. Open Forum Infect Dis. 2014;1(3):ofu103. https://doi. org/10.1093/ofid/ofu103.

24. McWhinney PH, Yates M, Prentice HG, et al. Infection caused by Mycobacterium chelonae: a diagnostic and therapeutic problem in the neutropenic patient, Clin Infect Dis, 1992, vol. 14(pg. 1208-1212).

25. Roukens AH, Mendels EJ, Verbeet NL, et al. Disseminated cutaneous mycobacterium chelonae infection in a patient with acute myeloid leukemia. Open Forum Infect Dis. 2014;1(3):ofu103. https://doi.org/10.1093/ofid/ofu103. Published 2014 Nov 26.

26. Doucette K, Fishman JA. Nontuberculous mycobacterial infection in hematopoietic stem cell and solid organ transplant patients. Clin Infect Dis. 2004;38:1428-39.

27. Doutre MS, Beylot C, Mougein J, et al. Cutaneous infection caused by Mycobacterium malmoense in a patient with myelodysplastic syndrome. J R Soc Med. 1993;86:110-1. 
28. Naorungroj S, Aiempanakit K. A study of cutaneous manifestations among febrile neutropenic patients: a five-year retrospective review in a single tertiary university hospital in Southern Thailand. J Am Acad Dermatol. 74(5):AB166. https://doi.org/10.1016/j.jaad.2016.02.653.

29. Feld R, Evans WK, DeBoer G. Herpes zoster in patients with small-cell carcimona of the lung receiving combined modality treatment. Ann Intern Med. 1980;93:282-3.

30. Mays SR, Bogle MA, Bodey GP. Cutaneous fungal infections in the oncology patient: recognition and management. Am J Clin Dermatol. 2006;7(1):31-43.

31. Pappas PG, et al. Clinical practice guideline for the management of candidiasis: 2016 update by the Infectious Diseases Society of America. Clin Infect Dis. 2016;62(4):e1-50.

32. Horn DL, et al. Epidemiology and outcomes of candidemia in 2019 patients: data from the prospective antifungal therapy alliance registry. Clin Infect Dis. 2009;48(12):1695-703.

33. Sipsas NV, et al. Candidemia in patients with hematologic malignancies in the era of new antifungal agents (2001-2007): stable incidence but changing epidemiology of a still frequently lethal infection. Cancer. 2009;115(20):4745-52.

34. Guarana M, Nucci M. Acute disseminated candidiasis with skin lesions: a systematic review. Clin Microbiol Infect. 2018;24(3):246-50.

35. Chen LY, et al. Associated clinical characteristics of patients with candidemia among different Candida species. J Microbiol Immunol Infect. 2013;46(6):463-8.

36. Nucci M, Anaissie E. Cutaneous infection by Fusarium species in healthy and immunocompromised hosts: implications for diagnosis and management. Clin Infect Dis. 2002;35(8):909-20.

37. Nucci M, Anaissie E. Fusarium infections in immunocompromised patients. Clin Microbiol Rev. 2007;20(4):695-704.

38. Hay RJ. Fusarium infections of the skin. Curr Opin Infect Dis. 2007;20(2):115-7.

39. Hayashida MZ, et al. Disseminated fusariosis with cutaneous involvement in hematologic malignancies: report of six cases with high mortality rate. An Bras Dermatol. 2018;93(5):726-9.

40. Gilbert DN, et al., editors. The Sanford guide to antimicrobial therapy 2018. Sperryville: Antimicrobial Therapy, Inc; 2018.

41. Bernardeschi $\mathrm{C}$, et al. Cutaneous invasive aspergillosis: retrospective multicenter study of the French invasive-aspergillosis registry and literature review. Medicine (Baltimore). 2015;94(26):e1018.

42. Patterson TF, et al. Invasive aspergillosis. Disease spectrum, treatment practices, and outcomes. I3 Aspergillus Study Group. Medicine (Baltimore). 2000;79(4):250-60.

43. Branscomb R. An overview of mucormycosis. Lab Med. 2002;33(6):453-5.

44. Patterson TF, et al. Practice guidelines for the diagnosis and management of aspergillosis: 2016 update by the Infectious Diseases Society of America. Clin Infect Dis. 2016;63(4):e1-e60.

45. Petrikkos G, et al. Epidemiology and clinical manifestations of mucormycosis. Clin Infect Dis. 2012;54(Suppl 1):S23-34.

46. Roden MM, et al. Epidemiology and outcome of zygomycosis: a review of 929 reported cases. Clin Infect Dis. 2005;41(5):634-53.

47. Ingram CW, et al. Disseminated zygomycosis: report of four cases and review. Rev Infect Dis. 1989;11(5):741-54.

48. Sun HY, Singh N. Mucormycosis: its contemporary face and management strategies. Lancet Infect Dis. 2011;11(4):301-11.

49. Chang P, Rodas C. Skin lesions in histoplasmosis. Clin Dermatol. 2012;30(6):592-8.

50. Lemos LB, Baliga M, Guo M. Blastomycosis: the great pretender can also be an opportunist. Initial clinical diagnosis and underlying diseases in 123 patients. Ann Diagn Pathol. 2002;6(3):194-203.

51. Miceli A, Krishnamurthy K. Blastomycosis. Treasure Island: StatPearls; 2018.

52. Wang S, et al. Dermatoscopic and clinicopathologic findings of cutaneous blastomycosis. J Am Acad Dermatol. 2015;73(5):e169-70.

53. DiCaudo DJ. Coccidioidomycosis: a review and update. J Am Acad Dermatol. 2006;55(6):929_ 42; quiz 943-5.

54. Christianson JC, Engber W, Andes D. Primary cutaneous cryptococcosis in immunocompetent and immunocompromised hosts. Med Mycol. 2003;41(3):177-88. 
55. Perfect JR, Bicanic T. Cryptococcosis diagnosis and treatment: what do we know now. Fungal Genet Biol. 2015;78:49-54.

56. Grunwald MR, McDonnell MH, Induru R, Gerber JM. Cutaneous manifestations in leukemia patients. Semin Oncol. 2016;43(3):359-65.

57. Kang YS, Kim HS, Park HJ, Lee JY, Kim HO, Cho BK, et al. Clinical characteristics of 75 patients with leukemia cutis. J Korean Med Sci. 2013;28(4):614-9.

58. Li AW, Yin ES, Stahl M, Kim TK, Panse G, Zeidan AM, et al. The skin as a window to the blood: cutaneous manifestations of myeloid malignancies. Blood Rev. 2017;31(6):370-88.

59. Benet C, Gomez A, Aguilar C, Delattre C, Vergier B, Beylot-Barry M, et al. Histologic and immunohistologic characterization of skin localization of myeloid disorders: a study of 173 cases. Am J Clin Pathol. 2011;135(2):278-90.

60. Cohen PR. Sweet's syndromeDOUBLEHYPHENa comprehensive review of an acute febrile neutrophilic dermatosis. Orphanet J Rare Dis. 2007;2:34.

61. Qiao J, Wang Y, Bai J, Wu Y, Fang H. Concurrence of Sweet's syndrome, pathergy phenomenon and erythema nodosum-like lesions. An Bras Dermatol. 2015;90(2):237-9.

62. Going JJ, Going SM, Myskow MW, Beveridge GW. Sweet's syndrome: histological and immunohistochemical study of 15 cases. J Clin Pathol. 1987;40(2):175-9.

63. Paydas S. Sweet's syndrome: a revisit for hematologists and oncologists. Crit Rev Oncol Hematol. 2013;86(1):85-95.

64. Kridin K, Cohen AD, Amber KT. Underlying systemic diseases in pyoderma Gangrenosum: a systematic review and meta-analysis. Am J Clin Dermatol. 2018;19(4):479-87.

65. Braswell SF, Kostopoulos TC, Ortega-Loayza AG. Pathophysiology of pyoderma gangrenosum (PG): an updated review. J Am Acad Dermatol. 2015;73(4):691-8.

66. Su WP, Davis MD, Weenig RH, Powell FC, Perry HO. Pyoderma gangrenosum: clinicopathologic correlation and proposed diagnostic criteria. Int J Dermatol. 2004;43(11):790-800.

67. Crowson AN, Mihm MC Jr, Magro C. Pyoderma gangrenosum: a review. J Cutan Pathol. 2003;30(2):97-107.

68. Loricera J, Calvo-Rio V, Ortiz-Sanjuan F, Gonzalez-Lopez MA, Fernandez-Llaca H, RuedaGotor J, et al. The spectrum of paraneoplastic cutaneous vasculitis in a defined population: incidence and clinical features. Medicine (Baltimore). 2013;92(6):331-43.

69. Greer JM, Longley S, Edwards NL, Elfenbein GJ, Panush RS. Vasculitis associated with malignancy. Experience with 13 patients and literature review. Medicine (Baltimore). 1988;67(4):220-30.

70. Garcia-Porrua C, Gonzalez-Gay MA. Cutaneous vasculitis as a paraneoplastic syndrome in adults. Arthritis Rheum. 1998;41(6):1133-5.

71. Miliszewski MA, Kirchhof MG, Sikora S, Papp A, Dutz JP. Stevens-Johnson syndrome and toxic epidermal necrolysis: an analysis of triggers and implications for improving prevention. Am J Med. 2016;129(11):1221-5.

72. Schwartz RA, McDonough PH, Lee BW. Toxic epidermal necrolysis: part I. Introduction, history, classification, clinical features, systemic manifestations, etiology, and immunopathogenesis. J Am Acad Dermatol. 2013;69(2):173, e1-13; quiz 85-6.

73. Roujeau JC, Kelly JP, Naldi L, Rzany B, Stern RS, Anderson T, et al. Medication use and the risk of Stevens-Johnson syndrome or toxic epidermal necrolysis. N Engl J Med. 1995;333(24):1600-7.

74. Macdonald JB, Macdonald B, Golitz LE, LoRusso P, Sekulic A. Cutaneous adverse effects of targeted therapies: part I: inhibitors of the cellular membrane. J Am Acad Dermatol. 2015;72(2):203-18; quiz 19-20.

75. Brazzelli V, Grasso V, Borroni G. Imatinib, dasatinib and nilotinib: a review of adverse cutaneous reactions with emphasis on our clinical experience. J Eur Acad Dermatol Venereol. 2013;27(12):1471-80. 